ECCOMAS

Proceedia
COMPDYN 2017

$6^{\text {th }}$ ECCOMAS Thematic Conference on Computational Methods in Structural Dynamics and Earthquake Engineering

M. Papadrakakis, M. Fragiadakis (eds.)

Rhodes Island, Greece, 15-17 June 2017

\title{
PREDICTION MODELS OF FREE-FIELD VIBRATIONS FROM RAILWAY TRAFFIC
}

\author{
Jens Malmborg ${ }^{1}$, Kent Persson ${ }^{1}$, Peter Persson ${ }^{1}$ and Lars Vabbersgaard Andersen ${ }^{2}$ \\ ${ }^{1}$ Lund University \\ Department of Construction Sciences \\ P.O. box 118 Lund, Sweden \\ \{jens.malmborg, kent.perssson, peter.persson\}@construction.lth.se \\ ${ }^{2}$ Aalborg University \\ Department of Civil Engineering \\ Thomas Mann vej 23, 9220, Aalborg, Denmark \\ la@civil.aau.dk
}

Keywords: Railway, vibration prediction, finite element model, ground vibration

Abstract. Many cities experience an increasing population leading to a need for urban densification. In these cities, unused land close to railways will have to be developed with new residential and office buildings. The infrastructural demand will also increase, resulting in heavily trafficked roads and railways close to where people work and live. Annoyance from trafficinduced vibrations and noise is expected to be a growing issue. To predict the level of vibration and noise in buildings caused by railway and road traffic, calculation models are needed.

In the present paper, a simplified prediction model is briefly described. This prediction model is based on the assumption that the ground and railway embankment can be described in an axisymmetric model, to provide the transfer functions between the track and the free-field. In the paper, the error that arise by assuming axisymmetric response is studied by comparing the response in a three-dimensional finite-element model. Transfer functions at several positions in the free-field are compared. 


\section{INTRODUCTION}

Many cities experience an increasing population leading to a need for urban densification. In these cities, unused land close to railways will have to be developed with new residential and office buildings. The infrastructural demand will also increase, resulting in heavily trafficked roads and railways close to where people work and live. Annoyance from traffic-induced vibrations and noise is expected to be a growing issue.

To predict the level of vibration and noise in buildings caused by, for example, railway and road traffic, calculation models are needed. To describe the problem, the models must include the source, medium, and receiver [1]. The source load is caused by the traffic, and in the medium the vibrations transmit to the receiver, which is where the vibrations are being observed.

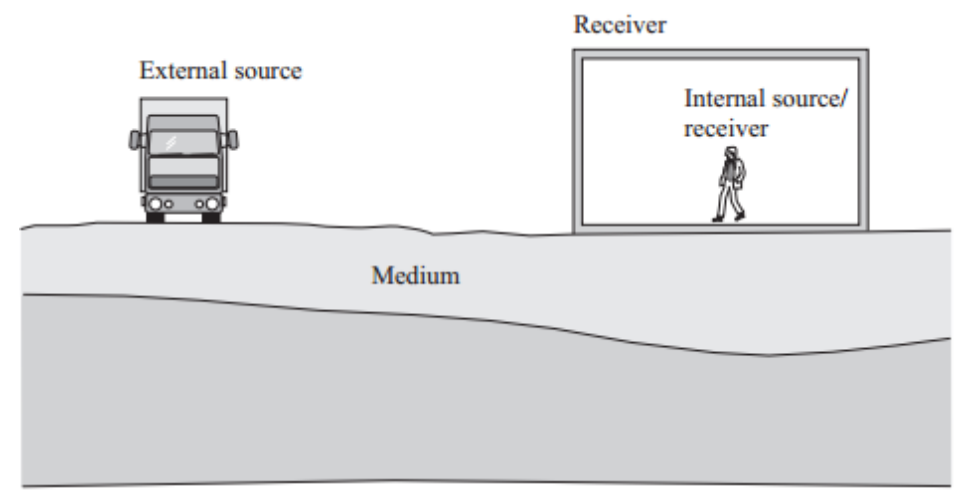

Figure 1: Sketch of the vibration-transmission process from source to receiver via the medium, from [1].

It is very difficult to accurately describe all mechanisms involved in the vibration generation from a passing train. With certain assumptions and simplifications, however, the prediction analysis can be divided into smaller independent steps. In one such simplified prediction model, briefly described in Section 1.1, the transfer functions between the railway track and the observation point in the free-field are estimated using an axisymmetric ground model. These transfer functions are then used together with estimations of the dynamic reaction forces of the railway track, calculated in a separate model, to provide the vibration response in the free-field.

In the present paper, the suitability of using an axisymmetric ground model to predict the vibrations in the free-field caused by a train running on an elevated embankment, is studied. This is investigated by using a 3D finite element (FE) model to calculate and compare the transfer functions obtained at several positions in the free-field at equal distances from the railway track, but at various angles from the embankment. The analyses are carried out for a few different soil layer depths.

\subsection{Background: A simplified prediction model}

The excitation caused by a moving train is often divided into a quasi-static part and a dynamic part, where the quasi-static part is simply the static deflection due to the weight of the train. The dynamic part is much more complex and involves several different phenomena that can be either periodic or random in nature, such as wheel flats, wheel/rail roughness, and varying sleeper/subgrade stiffness [2]. Track unevenness (roughness) is one of the main contributing factors to the vibrations generated by railway traffic, especially at lower speeds. When the train passes over the track irregularities, the contact force under each wheel varies with time, leading to a dynamic excitation of the ground. The properties of the ground, such as mechanical properties and stratification, are important factors for how the vibrations generated at the track are transferred to the observation point. 
If the soil is assumed to behave linearly elastic, and it is assumed that the wave propagation in the embankment does not influence the dynamic contact forces between the rail and the train wheels, a particularly simplistic approach can be established for estimating the vibrations generated in the free field due to a passing train.

A two-dimensional (2D) model consisting of a simplified track and vehicle representation is used for estimating the dynamic forces acting on each sleeper due to a train passing at a specific speed. These forces are subsequently used together with transfer functions, between each sleeper and the receiver (observation point) in the free field. By summing the response, from each individual sleeper, the total response at the observation point can be obtained.

A vehicle model, adopted here, that is frequently reported in the literature is that of a simple 10 degrees-of-freedom system consisting of springs, viscous dampers, lumped masses and rigid connections, shown schematically in Figure 1. The element coefficients can be adjusted to fit many different train types, as shown in [3]. Also adopted here, is a 2D model of a ballasted railway track with discrete sleepers. The model consists of a single rail, rail pads, sleepers, ballast and subsoil, modelled using beam elements, springs, viscous dampers and lumped masses, as shown in Figure 1.
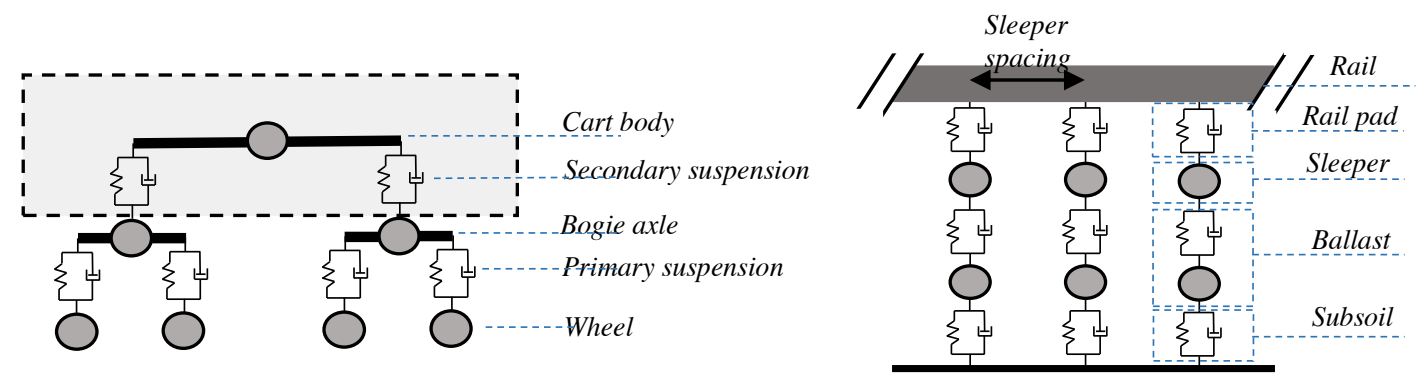

Figure 1: 2D models of train cart and railway track.

Track irregularities are often specified in terms of a power-spectral-density (PSD) spectrum, characterizing the unevenness in a statistical sense by decomposing the irregularities into power (variance) at different wavenumbers. In order to use such a spectrum in the spatial domain, a spatial realization is obtained by dividing the spectrum into an appropriate number of discrete wavenumbers, assigning a random phase to each wavenumber, and performing an inverse Fourier transform. Such a realization is incorporated into the track model above by changing the vertical coordinates of the beam nodes accordingly. A comprehensive list of different PSDs available for track irregularities is presented in [3].

The vehicle and track models are combined by letting the vehicle run over the track at a specific speed and enforcing some contact condition between the wheel mass elements and the rail beam elements. Due to the irregularities in the track and the varying flexibility attributable to the discrete supports (sleepers), the magnitude of the contact forces between the wheel and rail will vary along the rail. The randomness in the contact forces causes the element forces in the rail pad/ballast/subsoil representations to be unique at each individual sleeper position.

Considering the soil as a linear time-invariant system (cf. Eq. (1)), where an input signal $w(t)$ is the force applied at a certain sleeper position, the output signal $y(t)$ is the (e.g. vertical) displacement in an observation point, the input-output relationship is defined by the convolution integral

$$
y(t)=h * w(t)=\int_{-\infty}^{\infty} h(t-\tau) \cdot w(\tau) d \tau
$$


where $h(t)$ is the impulse response of the system. According to the convolution theorem, the time domain convolution integral turns into a simple multiplication in the frequency domain (if $h$ and $w$ are $L^{1}$ integrable)

$$
Y(f)=\mathcal{F}(y(t))=\mathcal{F}(h * w)=\mathcal{F}(h) \cdot \mathcal{F}(w)=H(f) \cdot W(f)
$$

Hence, the output signal is then obtained by an inverse Fourier transform,

$$
y(t)=\mathcal{F}^{-1}(H \cdot W)
$$

The Fourier transform of the impulse response is recognized as the transfer function (Frequency response function, FRF)

$$
\mathcal{F}(h(t))=H(f)
$$

Using the principle of superposition, the total response in a certain point is obtained as the sum of the responses from each excitation. This means that the response in an observation point $O$ in the free-field is obtained by calculating and summing the responses in the point $O$ due to the excitations at each individual sleeper position. Hence, the transfer functions to the observation point $O$, from all sleepers $s_{k}$ that are close enough to have any significance, are required (Figure 2).

The transfer functions can be estimated by various methods and models of different complexity; e.g. 2D or 3D FE models in either time- or frequency domain, or the so called domain transformation method (DTM) in which the wave propagation is analyzed using a semi-analytical approach in the frequency wavenumber domain [4]. Especially under axisymmetric conditions, both the FE method and the DTM can provide the transfer functions between the source and a receiver at a large number of distances, very quickly compared to large 3D FE models. However, even in cases where the soil is horizontally stratified, the railway track is usually placed on an embankment somewhat elevated from the surrounding ground surface, thereby making the suitability of an axisymmetric analysis approach less certain.

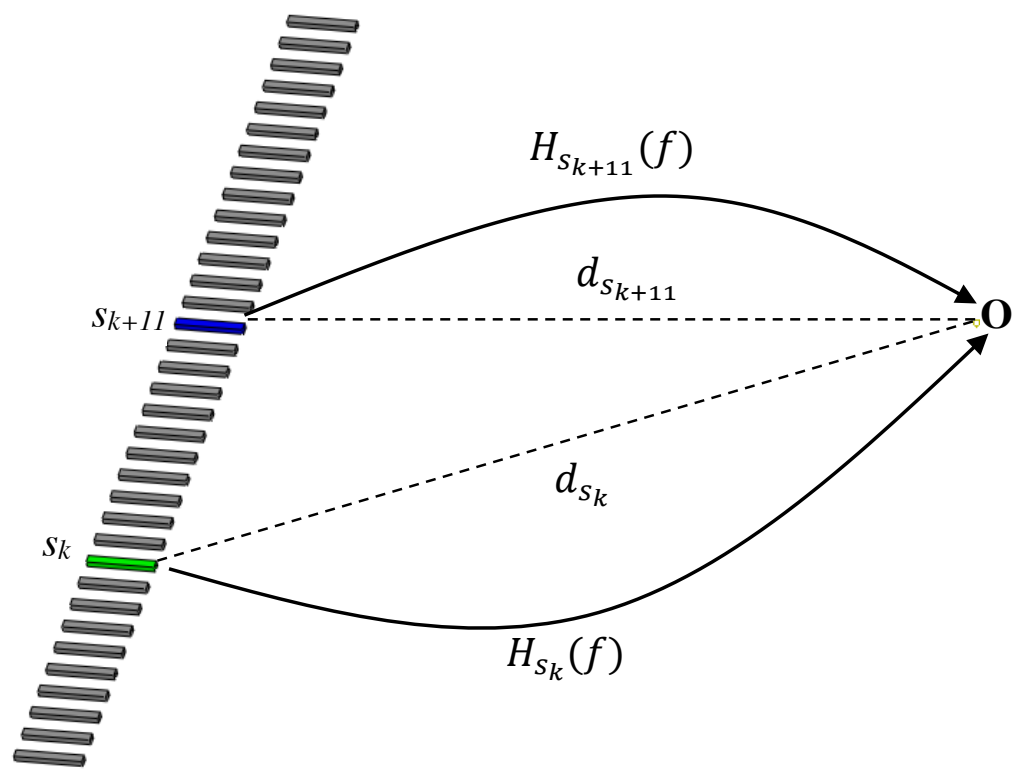

Figure 2: A transfer function $H(f)$ between each sleeper and the observation point in the free-field is needed. 


\section{A THREE-DIMENSIONAL GROUND MODEL}

The effect of the embankment on the transfer functions to the free-field is studied by use of a 3D FE model. The analysis is carried out in the commercial software Abaqus [5]. The model consists of a $75 \mathrm{~m}$ stretch of the railway embankment and the surrounding soil, see Figure 3 . On top of the embankment, one sleeper is modelled, on which a surface load is applied to a small part representing the area of interaction with the rail. Two symmetry planes exist.

Isoparametric second-order solid brick-elements with reduced integration are employed. To avoid waves reflecting back into the model at the boundaries, elements that implement viscous absorbing boundaries as proposed by Lysmer and Kuhlemeyer [6] are utilized. At these boundaries "tuned" dashpots are introduced that are completely effective for orthogonally impinging body waves, whereas other waves are only partially absorbed. However, these elements are usually deemed fit for engineering purposes, provided that they are placed sufficiently far from the region of interest and the damping in the medium is fairly high.

The ground model consists of one soil layer on top of bedrock. The depth of the soil layer is varied from 10 to 20 meters. The bedrock is modelled as a $20 \mathrm{~m}$ deep layer with viscous absorbing boundaries at the base. The total height of the embankment is $1 \mathrm{~m}$, including $0.3 \mathrm{~m}$ of ballast. The width is $6 \mathrm{~m}$ at the top and $10 \mathrm{~m}$ at the bottom, of which only half is modelled due to symmetry.

The soil and bedrock material parameters are presented in Table 1.

\begin{tabular}{lllll}
\hline & Soil & Bedrock & Embankment & Ballast \\
\hline Youngs modulus $(\mathrm{MPa})$ & 500 & 10,000 & 500 & 110 \\
Poisson's ratio $(-)$ & 0.48 & 0.4 & 0.48 & 0.36 \\
Mass density $\left(\mathrm{kg} / \mathrm{m}^{3}\right)$ & 2100 & 2500 & 2100 & 1550 \\
Damping ratio $(\%)$ & 3.5 & 2.0 & 3.5 & 3.5 \\
\hline
\end{tabular}

Table 1: Material properties used in the model

The Rayleigh wave speed in the soil material is approximately $280 \mathrm{~m} / \mathrm{s}$. Hence, the shortest wavelength is approximately $280 / 60=4.7 \mathrm{~m}$ at $60 \mathrm{~Hz}$. The approximate element size in the soil is $1.4 \mathrm{~m}$ within a radius of $50 \mathrm{~m}$; outside that radius, the element size is gradually increased up to a size of $4 \mathrm{~m}$. The resulting 7-9 nodes per wavelength was assumed to properly resolve a Rayleigh wave. Three second-order element edges contain seven nodes, meaning that the minimum wavelength is then approximately $3 \cdot 1.4=4.2 \mathrm{~m}$, which is approximately the wavelength of a Rayleigh wave at $66 \mathrm{~Hz}$. 


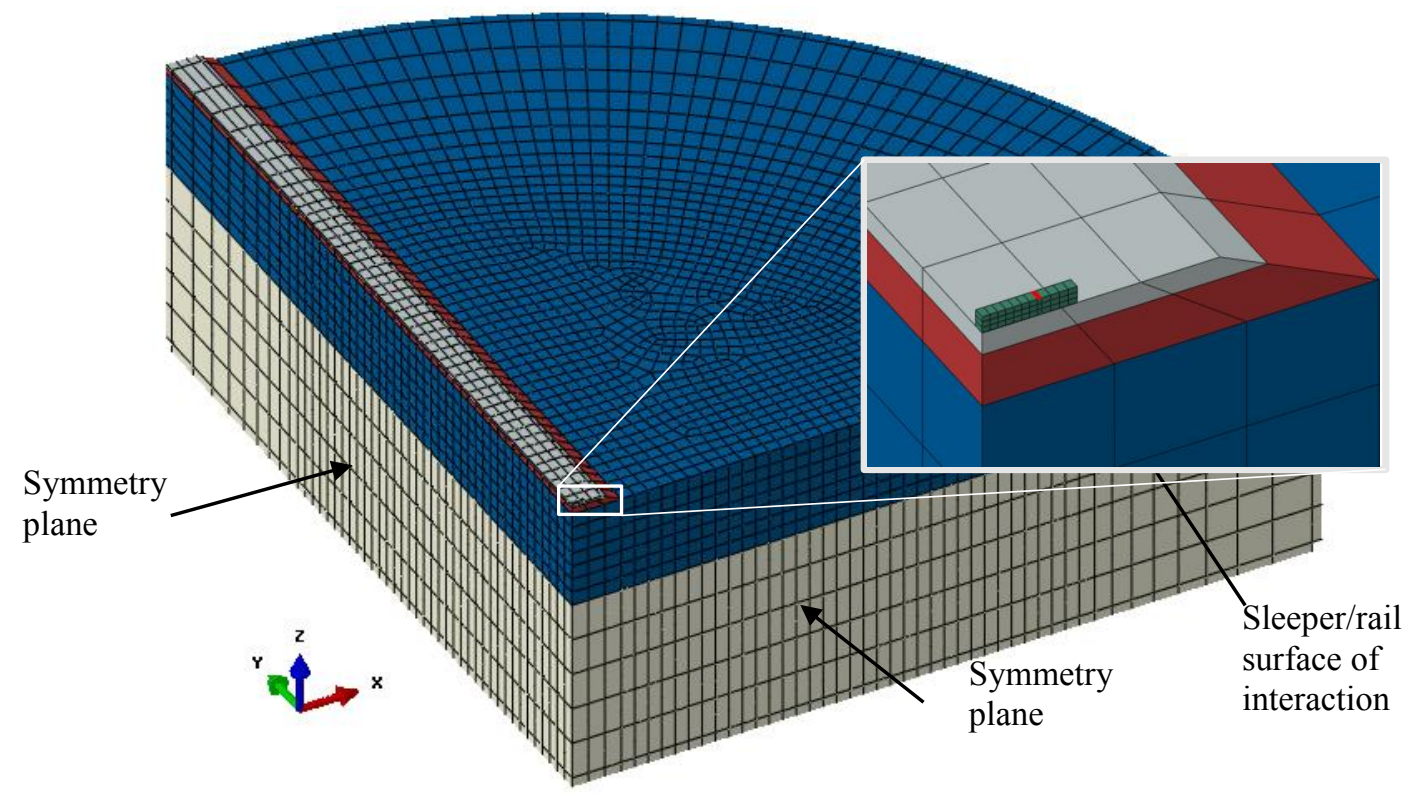

Figure 3: FE model used in this study

A steady-state analysis is performed in the frequency range 5-60 Hz, where a surface load is applied to the area of the sleeper representing the interface with the rail. The surface load is chosen arbitrarily to $10 \mathrm{kPa}$. The loaded area is $0.075 \mathrm{~m}^{2}$ and due to the two symmetry planes the total load is $10 \times 0.075 \times 4=300 \mathrm{~N}$. A steady state solution is found to the equation of motion

$$
\mathbf{M U ̈}+\mathbf{C} \dot{U}+\mathbf{K U}=\mathbf{P} \mathrm{e}^{\mathbf{i} \omega \mathbf{t}}
$$

where $\mathbf{M}$ is the mass matrix, $\mathbf{C}$ is the damping matrix, $\mathbf{K}$ is the stiffness matrix, and $\mathbf{P}$ is the load vector. $\mathbf{U}$ is the sought displacement vector.

With structural damping $\eta$, assuming a solution of the form $\boldsymbol{U}=\boldsymbol{u}^{*} e^{i \boldsymbol{t} t}$ gives

$$
\left(-\omega^{2} \mathbf{M}+\mathrm{i} \omega \mathbf{C}+(1+i \eta) \mathbf{K}\right) \mathbf{u}^{*} e^{\mathbf{i} \omega \mathbf{t}}=\mathbf{P} \mathrm{e}^{\mathbf{i} \boldsymbol{\omega t}}
$$

With $\mathbf{D}(\omega)=-\omega^{2} \mathbf{M}+\mathrm{i} \omega \mathbf{C}+(1+i \eta) \mathbf{K}$, the displacements are found as

$$
\mathbf{u}^{*}=\mathbf{D}^{-1}(\omega) \mathbf{P}
$$

The vertical response is studied in five groups of points, where the points in each group are located at equal distances from the load, but at different angles from the global $x$-axis which is perpendicular to the track. The distances are 10,20,30,40, and $50 \mathrm{~m}$ from the load. The points are located at intervals of $10^{\circ}$ to the global $x$-axis. The maximum angle for each distance is limited so that only points outside the embankment are studied, see Figure 4. 


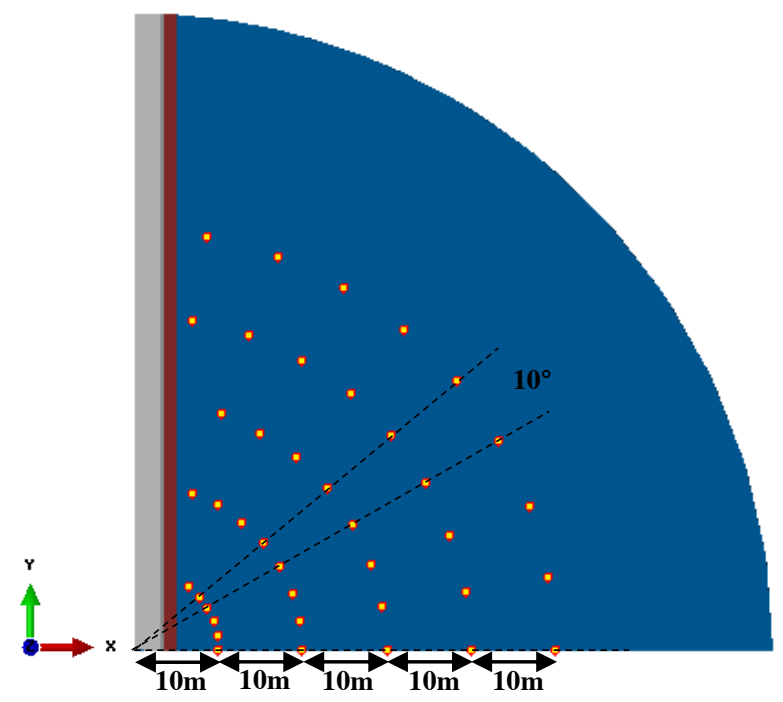

Figure 4: Points on the ground surface where the response is extracted. The points are located at 10, 20, 30, 40 and 50 meters from the load. The maximum angle for each distance is $50^{\circ}, 70^{\circ}, 70^{\circ}, 80^{\circ}$, and $80^{\circ}$, respectively.

Generally, the FE model does not have nodes at these exact points. Therefore, the results are extracted for all the nodes in the element surrounding the sought point. The element shape functions are then used to map the point to the element parent domain, as well as to interpolate the response using the element nodal values.

Figure 5 (left) shows the envelopes for each distance, where the thickness of the envelope indicates some deviation from axisymmetric behavior. In Figure 5 (right), the absolute values of the transfer functions are shown for all points, for the case with a soil layer depth of $10 \mathrm{~m}$.

The absolute values of the transfer functions are shown for all points in Figure 6 grouped by the distance from the load, and normalized to the value at the point at $0^{\circ}$.
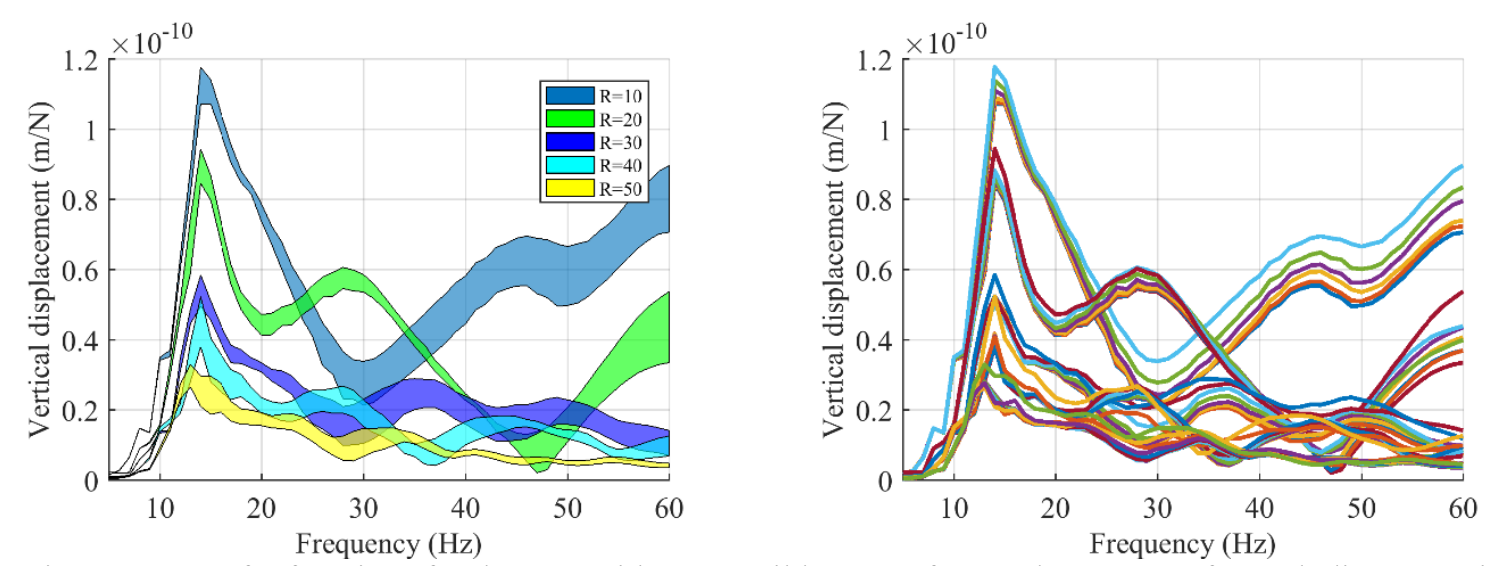

Figure 5: Transfer functions for the case with $10 \mathrm{~m}$ soil layer. Left: Envelope curves for each distance. Right: Absolute value of the transfer functions for all points. 


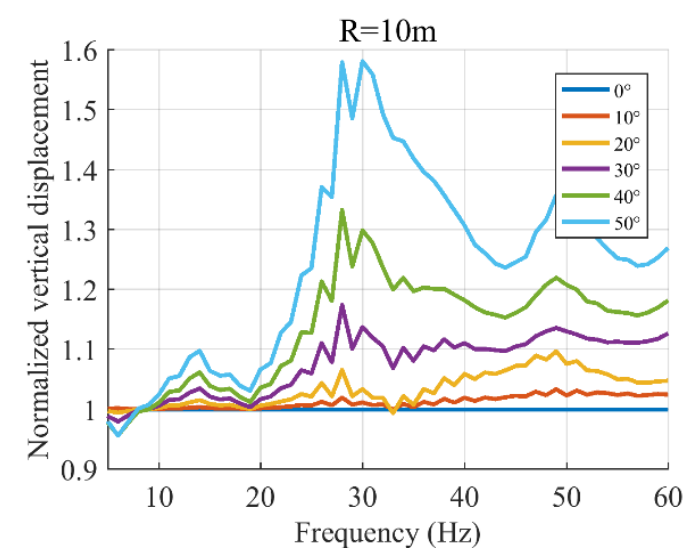

(a)

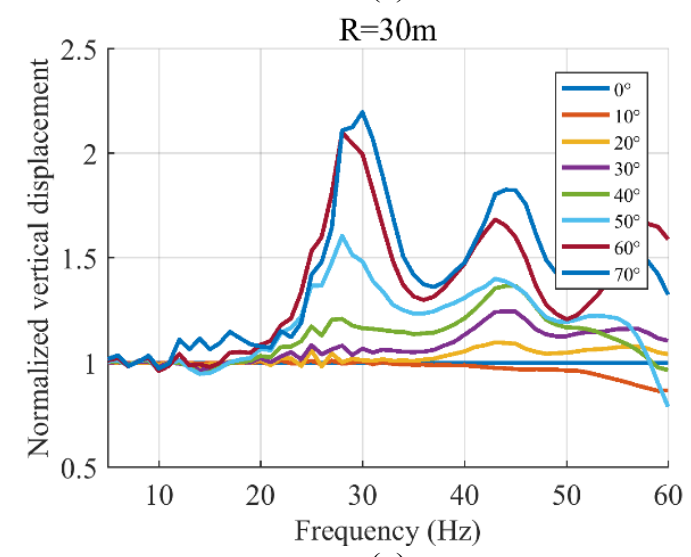

(c)

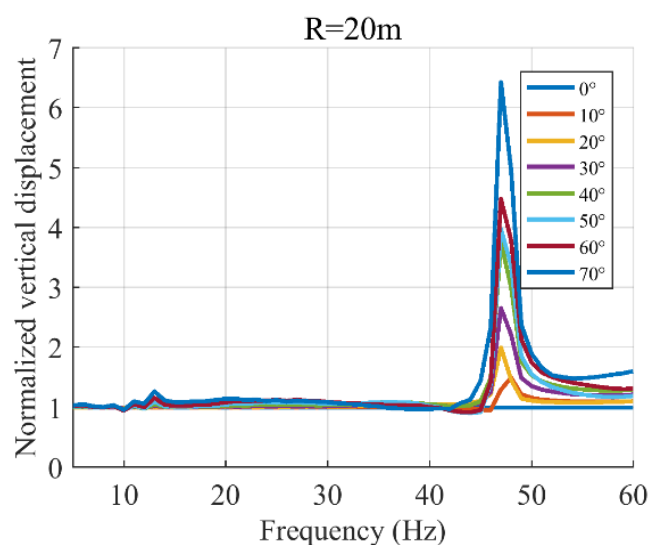

(b)

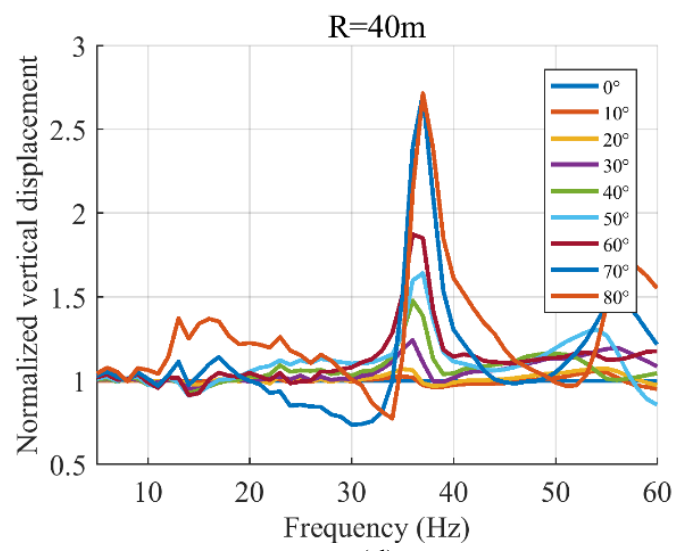

(d)

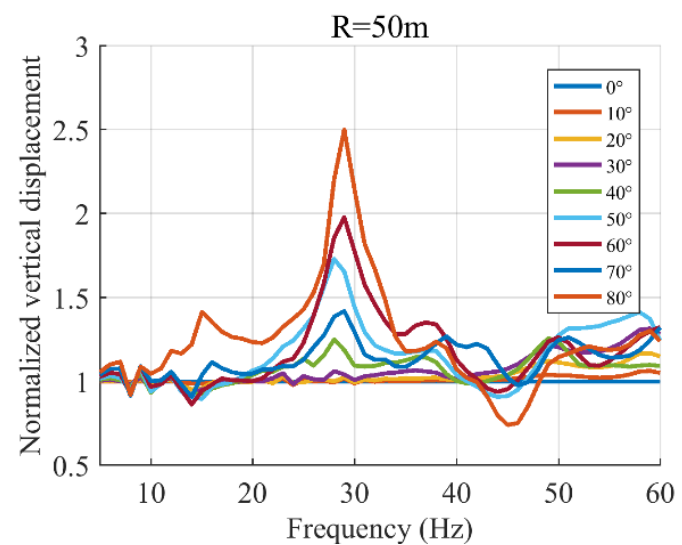

(e)

Figure 6: Absolute value of transfer function normalized against the values for the point at $0^{\circ}$ for all points at (a) $10 \mathrm{~m}$, (b) $20 \mathrm{~m}$, (c) $30 \mathrm{~m}$, (d) $40 \mathrm{~m}$, (e) $50 \mathrm{~m}$ from the load.

As shown in Figures 5 and 6, the difference between points at equal distance from the load can become quite large, especially when comparing the point at $0^{\circ}$ (perpendicular to the embankment) and the points at higher angles (i.e. points that are relatively close to the embankment). The analysis of the case with a $20 \mathrm{~m}$ deep soil layer shows similar results and are therefore not presented here.

However, considering a fixed observation point in the free field, the relative importance of the transfer functions for points on the track that are located at larger angles between the observation point and a line perpendicular to the track, is in general naturally lower, since the former points are located farther away from the observation point. In order to quantify this effect, the transfer functions have been extracted for a number of points, equivalent to traversing along the 
track and extracting the transfer function between each sleeper and a fixed observation point. A sleeper spacing of 0.6 meters is assumed. This is carried out for a stretch of $49.8 \mathrm{~m}$. This provides $49.8 / 0.6+1=84$ points which, due to symmetry, can be "mirrored" in order to provide transfer functions for $84+83=167$ sleepers. The absolute values of the transfer functions are summed. For comparison, an equal number of transfer functions is extracted for points at corresponding distances but all positioned on the global $x$-axis, i.e. perpendicular to the embankment, thus providing an "axisymmetric equivalent". The absolute value of these are also summed. This is carried out for three observation points, located 10, 20 and $30 \mathrm{~m}$ from the track. The points are shown in Figure 7 for the case of an observation point $30 \mathrm{~m}$ from the track. The results are shown in Figure 8.

The comparison shows that the summed transfer functions differ only slightly, indicating that the error in disregarding the asymmetry and only using transfer functions from points located perpendicular to the embankment, is limited.

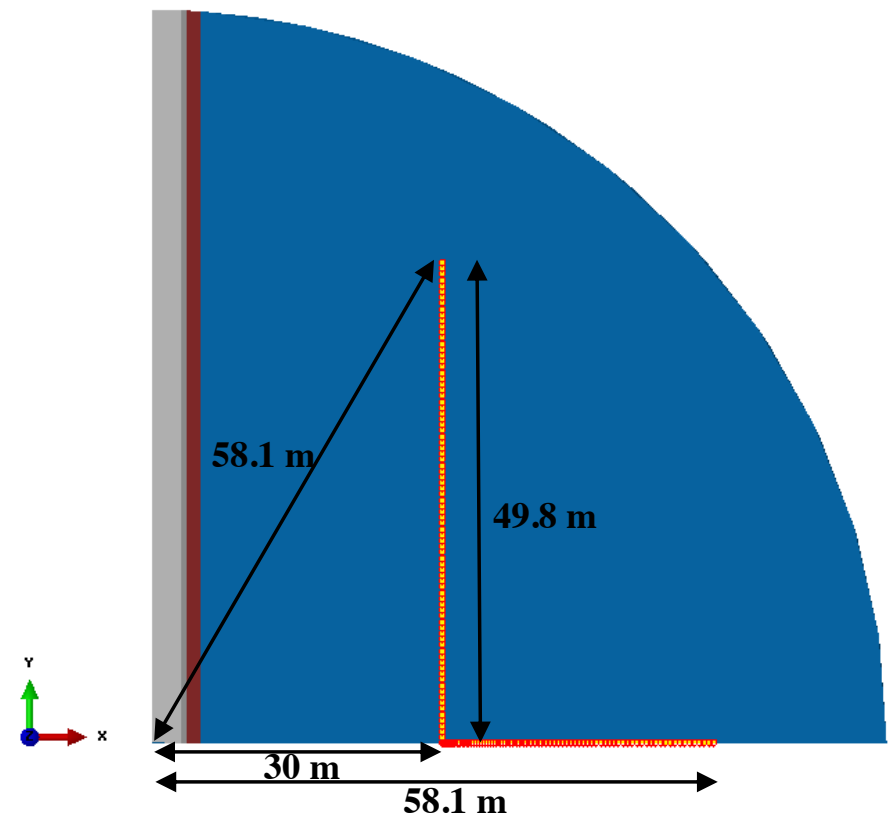

Figure 7: Points on the ground surface used for extracting transfer functions for 84 different sleepers, for an observation point 30 meter from mid-embankment. 


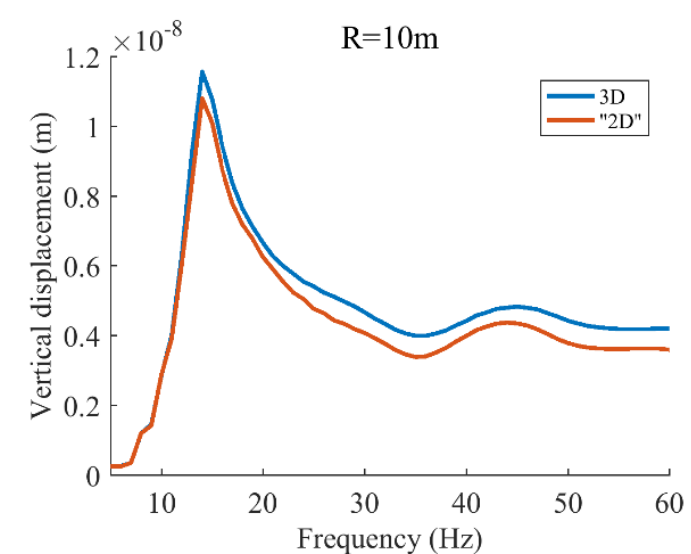

(a)

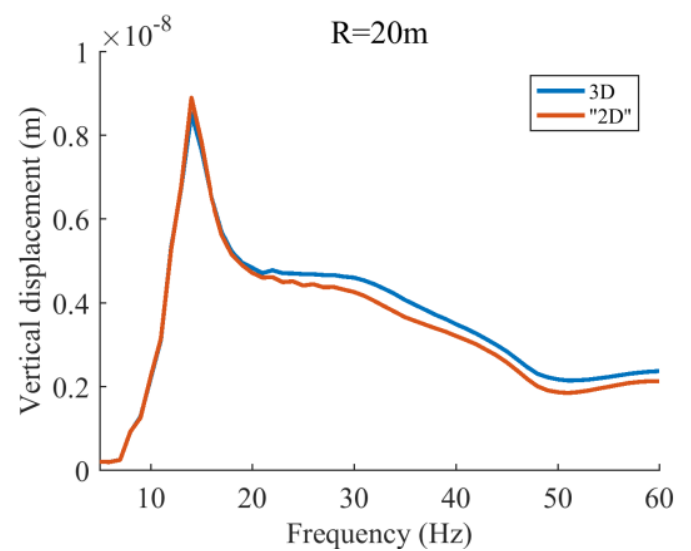

(b)

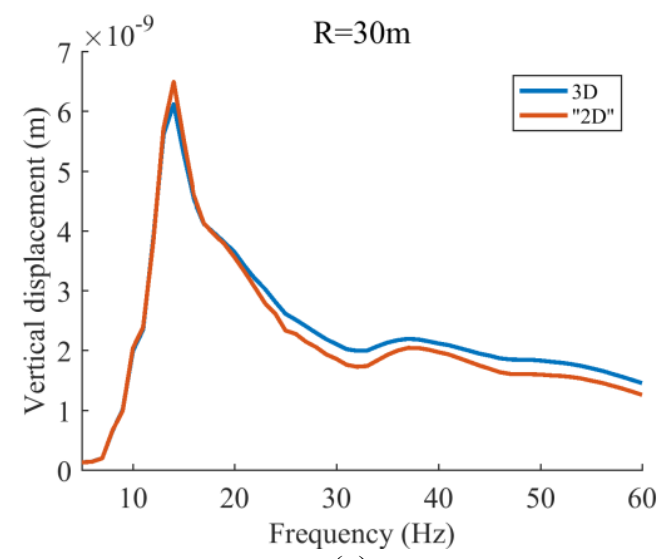

(c)

Figure 8: Comparison of the absolute sum of transfer functions for 167 sleepers when considering or disregarding (3D / "2D”) the asymmetry in displacement amplitudes relative the global $x$-axis.

\section{CONCLUSIONS}

In the present paper, the effect of the embankment on the transfer functions from a railway track to an observation point in the free-field has been studied by comparing the vertical displacement for points at equal distance from the load, but at different angles to the embankment, for loads in the frequency range 5-60 Hz. The most important findings are:

- In the studied cases, the vertical displacement for different points in the free-field, with equal distance to the load, vary significantly depending on the angle to the embankment. In general, the displacement increases for points that are closer to the embankment, i.e. for increasing angles.

- Evaluating the free-field response due to a train passage using a simplified approach such as proposed in Section 1.1, an axisymmetric soil model can probably still be useful, as long as it provides the same response as a 3D model does for points perpendicular to the embankment. The reason is that in general, the relative importance of sleepers forming large angles to the observation point is low when compared to sleepers close to perpendicular to the track, due to the increased distance to the observation point.

- To draw any general conclusions a larger parameter study is required, where the geometrical shape and the stiffness of the embankment is varied, as well as the properties of the soil stratification. 


\section{ACKNOWLEDGMENTS}

The research was carried out in the framework of the project "Urban Tranquility" under the Interreg V programme. The authors of this work gratefully acknowledge the European Regional Development Fund for the financial support.

\section{REFERENCES}

[1] P. Persson, Vibrations in a built environment - Prediction and Reduction. Doctoral Thesis, Report TVSM-1026, Lund University, Sweden, 2016.

[2] G. Lombaert, G. Degrande, S. François, D.J. Thompson, Ground-Borne Vibration due to Railway Traffic: A Review of Excitation Mechanisms, Prediction Methods and Mitigation Measures, Proceedings of the 11th International Workshop on Railway Noise, Uddevalla, Sweden, 9-13 September 2013.

[3] D. Cantero, T. Arvidsson, E. Obrien, R. Karoumi, Train-track-bridge modelling and review of parameters, Structure and Infrastructure Engineering, 12:(9), 1051-1064, 2016, DOI: $10.1080 / 15732479.2015 .1076854$.

[4] L.V. Andersen, J. Clausen, Efficient Modelling of Wind Turbine Foundations. In R. Carriveau (Ed.), Fundamental and Advanced Topics in Wind Power (Chapter 6, pp. 115-174), 2011. Rijeka, Croatia: INTECH open. DOI: 10.5772/731

[5] Dassault Systémes. Abaqus 6.14. Documentation, USA, 2014.

[6] J. Lysmer, R.L. Kuhlemeyer, Finite dynamic model for infinite media, Journal of Engineering Mechanics (ASCE), 859-877, 1969. 